\title{
The Effect of Ageing on Optimal Integration of Conflicting and Non-conflicting Visual-haptic Stimuli
}

DOI:

10.1163/22134808-20191409

\section{Document Version}

Accepted author manuscript

Link to publication record in Manchester Research Explorer

\section{Citation for published version (APA):}

Couth, S., Poole, D., Gowen, E., Champion, R. A., Warren, P. A., \& Poliakoff, E. (2019). The Effect of Ageing on Optimal Integration of Conflicting and Non-conflicting Visual-haptic Stimuli. Multisensory Research.

https://doi.org/10.1163/22134808-20191409

\section{Published in:}

Multisensory Research

\section{Citing this paper}

Please note that where the full-text provided on Manchester Research Explorer is the Author Accepted Manuscript or Proof version this may differ from the final Published version. If citing, it is advised that you check and use the publisher's definitive version.

\section{General rights}

Copyright and moral rights for the publications made accessible in the Research Explorer are retained by the authors and/or other copyright owners and it is a condition of accessing publications that users recognise and abide by the legal requirements associated with these rights.

\section{Takedown policy}

If you believe that this document breaches copyright please refer to the University of Manchester's Takedown Procedures [http://man.ac.uk/04Y6Bo] or contact uml.scholarlycommunications@manchester.ac.uk providing relevant details, so we can investigate your claim.

\section{OPEN ACCESS}


The Effect of Ageing on Optimal Integration of Conflicting and Non-conflicting Visualhaptic Stimuli

Samuel Couth ${ }^{1 *}$, Daniel Poole ${ }^{2}$, Emma Gowen ${ }^{2}$, Rebecca A. Champion ${ }^{2}$, Paul A. Warren ${ }^{2}$ and Ellen Poliakoff $^{2}$

${ }^{1}$ Division of Human Communication, Development and Hearing

${ }^{2}$ Division of Neuroscience and Experimental Psychology

Faculty of Biology, Medicine and Health, University of Manchester, UK

${ }^{*}$ Corresponding author: Samuel Couth

Email: Samuel.couth@manchester.ac.uk

Postal address: A3.16 Ellen Wilkinson Building, University of Manchester, Oxford Road, Manchester, UK, M13 9PL

This is a pre-copyedited, author-produced PDF of an article accepted for publication in Multisensory Research (Brill) following peer review. This version has been produced ahead of publication. The final published version of the article Couth et al. (2019) will be available online at https://brill.com/view/journals/msr/msr-overview.xml. 


\begin{abstract}
Multisensory integration typically follows the predictions of a statistically optimal model whereby the contribution of each sensory modality is weighted according to its reliability. Previous research has shown that multisensory integration is affected by ageing, however it is less certain whether older adults follow this statistically optimal model. Additionally, previous studies often present multisensory cues which are conflicting in size, shape or location, yet naturally occurring multisensory cues are usually non-conflicting. Therefore, the mechanisms of integration in older adults might differ depending on whether the multisensory cues are consistent or conflicting. In the current experiment, young $(n=21)$ and older $(n=30)$ adults were asked to make judgements regarding the height of wooden blocks using visual, haptic or combined visual-haptic information. Dual modality visual-haptic blocks could be presented as equal or conflicting in size. Young and older adults' size discrimination thresholds (i.e. precision) were not significantly different for visual, haptic or visual-haptic cues. In addition, both young and older adults' discrimination thresholds and points of subjective equality did not follow model predictions of optimal integration, for both conflicting and non-conflicting cues. Instead, there was considerable between-subject variability as to how visual and haptic cues were processed when presented simultaneously. This finding has implications for the development of multisensory therapeutic aids and interventions to assist older adults with everyday activities, where these should be tailored to the needs of each individual.
\end{abstract}

\title{
Keywords
}

Multisensory integration; visual-haptic; ageing; maximum likelihood estimate; therapeutic interventions 


\section{Introduction}

A coherent percept of our environment is formed by the merging of multiple sources of sensory information from a variety of different modalities, such as vision, touch and audition (Stein and Meredith, 1993). It is thought that multisensory signals are merged via different neural processes depending on the nature of the information provided by those signals (for a review see Ernst and Bülthoff, 2004). Briefly, 'sensory combination' describes interactions between non-redundant sensory signals, such that each modality provides distinct information to disambiguate a noisy sensory scene or to enrich the environmental percept. By contrast, 'sensory integration' describes interactions between redundant signals, such that each modality provides information that is in the same units and is about the same aspect of an environmental property, such as the size, orientation or location of an object, with the purpose of increasing the reliability of the percept.

The effects of ageing on the individual senses are well documented, showing age-related sensory decline for essentially all modalities (see Nusbaum, 1999), and more recently it has been shown that multisensory processing is also affected by ageing (de Dieuleveult et al., 2017; Mozolic et al., 2012). With regards to 'sensory combination', several studies have tested young and older adults on a "moving room" paradigm which involves manipulating visual cues to give a false-sense of selfmotion, and thus requires vestibular cues to resolve the ambiguity (e.g. Mahboobin et al., 2005; Ravaioli et al., 2005). The results commonly show a greater amount of postural instability in older adults compared to young, suggesting that older adults are over-reliant on visual cues, and fail to use vestibular or proprioceptive cues to disambiguate the sense of self-motion, which could be fundamental to trips and falls in older adults (Eikema et al., 2012; Jeka et al., 2006; Sundermier et al., 1996). With regards to 'sensory integration' (the focus of the study presented here), experiments have demonstrated relatively faster detection of multisensory cues in older adults (Couth et al., 2018a; Hugenschmidt et al., 2009; Laurienti et al., 2006; Mahoney et al., 2011; Peiffer et al., 2007), as well as increased temporal (Poliakoff et al., 2006b; Setti et al., 2011a, 2011b; Virsu et al., 2003) and spatial (Couth et al., 2016; Mahoney et al., 2014b; Poliakoff et al., 2006a) windows in which multisensory cues are integrated, compared to young adults. This tendency to integrate spatially and temporally discrepant information could be a beneficial compensatory mechanism to counteract poorer unisensory (Cienkowski and Carney, 2002; Dumas et al., 2016; Laurienti et al., 2006) or physical functioning (Mahoney et al., 2015, 2014a; Teramoto et al., 2017), or could be a detrimental effect due to poorer selective attention (Poliakoff et al., 2006a, 2006b). Nevertheless, while previous studies have explored how and why multisensory integration might be affected by increasing age, the process by which individual sensory inputs are integrated into a coherent percept in older adults 
is much less understood. This could have implications for the design of therapeutic aids, where previous research has suggested capitalising on enhanced multisensory integration in older adults to develop tools to assist with activities of daily living (e.g. Mahoney et al., 2014b).

It has been proposed that multisensory integration is statistically optimal (Ernst and Banks, 2002; Rohde et al., 2016). Under optimal integration the individual sensory cues should be combined to create a maximum likelihood estimate (MLE) using a weighted average (see equation 1 in Methods), where weights are determined by the precision of the unisensory cues. The subsequent estimate is statistically optimal in having the maximum precision possible and higher precision than either unisensory cue. Optimal integration has been tested empirically in experiments which require participants to make a judgement about the physical properties of an object (e.g. size, shape, orientation) using unisensory cues only (e.g. visual or haptic) or multisensory cues (e.g. visualhaptic), and then comparing measured multisensory performance to an MLE prediction of multisensory performance. For example, Ernst and Banks (2002) asked participants to make comparative height judgements of virtual reality bars using visual, haptic or combined visual-haptic cues. In dual modality conditions, visual-haptic stimuli were unequal in height (i.e. in conflict), enabling the authors to determine whether the participants' point of subjective equality (PSE; i.e. the smallest height difference between two comparative stimuli where participants are unable to tell the difference between them) was biased more towards visual or haptic cues, and thus indicated which cue was given more weight in combined visual-haptic conditions. The results showed that dual modality visual-haptic variance was lower (i.e. more precise) than either of the unimodal stimuli, and was consistent with MLE predicted variances. Moreover, visual weights were higher than haptic (see equation 2 in Methods), and visual-haptic PSEs were biased towards visual information, consistent with MLE predicted PSEs (see equation 3 in Methods). Performance of young adults on multisensory tasks has also been shown to be consistent with the optimal model of integration when making judgements in experiments which use different stimulus properties (e.g. spatial location) and different sensory pairings (e.g. audiovisual) (Alais and Burr, 2004; Drewing and Ernst, 2006; Hillis et al., 2004; for a review see Van Dam et al., 2014).

Gori and colleagues (2008) have since developed a simple version of the visual-haptic size discrimination task used by Ernst and Banks (2002) - that does not require computer generated stimuli - to determine the developmental trajectory of optimal integration. The authors demonstrated that performance was not well predicted by the MLE model until the age of 10 . For children aged 5 and 6, performance in dual modality conditions was biased towards the haptic cue, despite showing lower variance (i.e. increased precision) for the visual cue. The authors proposed 
that sensory systems need to be continuously recalibrated during development to take into account physical growth of the body and sensory organs. In this case, it may be important to prioritise calibration rather than optimally integrate the senses.

Applying these findings to older adults; age-related deterioration to the senses could lead to perceptual uncertainty, and thus sensory systems may also need to be continuously recalibrated. Therefore, it is conceivable that multisensory integration would not be optimal for older adults either. There have been a small number of studies which have investigated the effects of ageing on optimal integration. For example, on a visual-haptic task which required participants to align a metal bar with the subjective vertical, performance was comparable between young and older adults, with both age groups up-weighting visual cues and following MLE predictions in multisensory conditions (Braem et al., 2014). Ramkhalawansingh and colleagues (2018) also showed that both young and older adults integrate congruent visual and vestibular cues in a statistically optimal way on a selfmotion perception task. However, when there was a conflict between visual and vestibular information, only young adults demonstrated reliability-based cue weighting for conflicting cues, whereas older adults' estimates were biased towards the less reliable visual cue. Similarly, Bates and Wolbers (2014) showed that, compared to young adults, older adults do not optimally integrate conflicting visual and self-motion information on a spatial navigation task. Ramkhalawansingh and colleagues (2018) proposed that older adults' tendency to integrate conflicting and incorrectly weighted visual cues may hinder performance on everyday tasks of daily living, such as mobility and driving.

Interestingly, several studies have shown that young adults do not always follow optimal multisensory integration either (Arnold et al., 2019; Billino and Drewing, 2018; Cellini et al., 2013; Gepshtein et al., 2005; Kuschel et al., 2010; Oruç et al., 2003; Poole et al., 2017; Rosas et al., 2005). Optimal integration may be dependent on the physical property which is being measured, for example integration of visual and haptic cues for the perception of softness (Cellini et al., 2013) and slant (Oruç et al., 2003) have been shown to deviate from optimal. Alternatively, cues which are spatially discrepant might not be optimally integrated (Gepshtein et al., 2005), whereby only spatially congruent cues should be optimally integrated to increase the precision of the multisensory estimate (Rahnev and Denison, 2018), as is consistent with the spatial rule of integration (Stein and Meredith, 1993). Instead, multisensory performance may follow a suboptimal model of integration for conflicting or discrepant cues, whereby combined cues are not weighted according to their reliability, and thus the precision of the multisensory estimate is not increased compared to unisensory (i.e. performance is less than optimal; Billino and Drewing, 2018; Kuschel et al., 2010). 
Alternatively, young adults may follow a stochastic cue-switching (SCS) model whereby sensory signals are not integrated, but rather judgements in multisensory conditions are made using only one of the unisensory cues (usually the more reliable), which can alternate from trial-to-trial (Kuschel et al., 2010; Landy and Kojima, 2001; Poole et al., 2017; Serwe et al., 2009). The precision of the SCS estimate is not increased compared to either of the unisensory cues since additional variability may be added in trials where less reliable cues are up-weighted.

Recently, Billino and Drewing (2018) used a length discrimination task with conflicting visual and haptic size information to explore whether young and older adults' visual-haptic judgements followed MLE (i.e. optimal), SCS or suboptimal predictions. Whilst multisensory performance was similar between groups, older adults' multisensory performance agreed with both MLE and suboptimal predictions, whereas young adults' performance was closer to suboptimal model predictions only, with less visual up-weighting than predicted by the MLE. The authors concluded that older adults may exploit available sensory information more efficiently than young adults, which might compensate for unisensory degradation. However, all comparison stimuli in this study were unisensory (haptic), which could require effortful visual-to-haptic matching in visual only and dual modality conditions, and could mean that participants relied more exclusively on haptic cues in dual modality conditions. Older adults in this study performed poorly in visual only conditions compared to haptic only or visual-haptic conditions, and equally well on haptic and visual-haptic conditions, meaning that measured multisensory performance was more consistent with model predictions (i.e. increased haptic weight). On the contrary, young adults performed equally well on visual and haptic only conditions (i.e. equal visual and haptic weights), but relied more on haptic stimuli in the dual modality conditions, and thus did not appear to perform in an optimal way. It may have been more (or equally) efficient to rely on unweighted cues in this task (i.e. suboptimal integration), or a haptic only cue, rather than attempt to optimally integrate these, whereby there is no opportunity for multisensory performance benefit relative to haptic only cues.

It is important to note that sensory conflicts are artificially created in laboratory tasks designed to test integration, and occur less commonly in the natural environment (although sensory signals are often ambiguous in the natural environment; see Ernst and Bülthoff, 2004). As such, participants may be aware of the sensory conflict which could encourage a conscious decision-making strategy when making judgements about conflicting stimuli (De Gelder and Bertelson, 2003). Previous studies which have only included conflicting cues may be limited in what they can reveal about how older adults weight and integrate multisensory cues, and so it is also important to understand how older adults weight and integrate non-conflicting sensory information. This could help to develop 
interventions which assist older adults in correctly integrating sensory cues in real-world nonconflicting environments.

In the current study, we aimed to determine how the process of statistically optimal multisensory integration is affected by ageing for conflicting and non-conflicting sensory cues. Furthermore, we aimed to determine whether MLE, SCS or suboptimal models provide the best prediction of multisensory performance, where previous studies have not assessed these alternative models for both conflicting and non-conflicting sensory cues (cf. Billino and Drewing, 2018; Ramkhalawansingh et al., 2018). We have recently developed a low-technology version of a visual-haptic size discrimination task (Poole et al., 2017), which we used here to compare performance of young and older adults on conflicting and non-conflicting multisensory conditions. This task is closely modelled on that used by Gori and colleagues (2008), which was found to be a suitable method for measuring optimal integration of visual-haptic cues in young adults.

\section{Methods}

Participants

Thirty older (female $n=16$; mean age $=73.2 \pm 6.1$ years; right handed $n=27$ ) and twenty-one young adults (female $n=12$; mean age $=21.8 \pm 6.0$ years; right handed $n=18$ ) took part in this experiment. Older adults were recruited from the local Manchester community. Young adults consisted of undergraduate students and volunteers recruited via the University of Manchester research volunteering website. All participants were screened to determine their suitability for inclusion in the study during a previous visit as part of a larger investigation. Participants were required to have equal to- or better than-6/12 (20/40) visual acuity (with or without correction) as assessed via the Snellen letter chart, indicating good acuity for all participants (Falkenstein et al., 2008; Kaiser, 2009). Older participants were screened for dementia using the Mini Mental State Examination (MMSE; score $\geq 24$ indicates normal cognition; Folstein et al., 1975). Older participants also completed the Berg Balance Scale to determine risk of falls, with all participants deemed to be at low-risk (score $\geq 41 / 56$ indicates low-risk; Berg, 1989). Participants had no history of any other neurological conditions (e.g. Parkinson's disease or neuropathy) or severe head injuries. The study was approved by the University of Manchester Research Ethics Committee in accordance with the Declaration of Helsinki 2013.

\section{Stimuli and Apparatus}

Stimuli consisted of wooden blocks mounted onto the front and back of an acrylic cartridge (see Gori et al., 2008; Poole et al., 2017). The blocks were all equal in width $(100 \mathrm{~mm})$ and depth $(10 \mathrm{~mm})$, but 
had variable height ranging from $48 \mathrm{~mm}$ to $62 \mathrm{~mm}$ in $2 \mathrm{~mm}$ increments (referred to herein as comparison stimuli; Fig. 1a). Block sizes were the same on the front and back of the cartridges. There were three additional cartridges; one with a $55 \mathrm{~mm}$ block on the front and back (referred to herein as the no-conflict standard), and two 'conflict' cartridges which had a $52 \mathrm{~mm}$ block at the front and $58 \mathrm{~mm}$ block at the back (referred to herein as conflict standard 1), or $58 \mathrm{~mm}$ block at the front and $52 \mathrm{~mm}$ at the back (referred to herein as conflict standard 2) (Fig. 1b). Blocks were positioned onto the cartridges so that the centre was always $110 \mathrm{~mm}$ from the top of the cartridge, thus changes in block height occurred in both up and down directions. An acrylic display stand was used which allowed the cartridges displaying the blocks to be easily placed and removed (Fig. 1c). The centre of the blocks was $280 \mathrm{~mm}$ from the surface of the desk when mounted onto the display stand.

Participants wore PLATO visual occlusion spectacles (Translucent Technologies Inc.) throughout the experiment. Between presentations, the spectacles were opaque, thus occluding vision. E-Prime (Psychology Software Tools) was used to trigger the spectacles to become transparent, thus allowing precise timing of visual input.

[Figure 1.]

Design

A two-interval forced choice design was implemented. For visual only and haptic only conditions (see Procedure below), participants were presented with the no-conflict standard $(55 \mathrm{~mm})$ block in one interval and a taller $(56,58,60,62 \mathrm{~mm})$ or shorter $(48,50,52,54 \mathrm{~mm})$ comparison block in the other interval. Participants were required to verbally indicate which of the intervals ("One" or "Two") contained the taller of the two blocks. The order of comparison stimuli was randomised, and the standard-comparison interval order was counterbalanced.

For the combined visual-haptic condition (referred to herein as dual condition) without conflicting stimuli, the experimental design was the same as the visual only and haptic only conditions. For the dual condition with conflicting stimuli, the no-conflict standard was replaced by either conflict standard $1(52 / 58 \mathrm{~mm})$ or conflict standard $2(58 / 52 \mathrm{~mm})$.

To gain a better estimate of discrimination thresholds and the point of subjective equality (PSE; i.e. the block size where participants were unable to tell the difference between the standard block and comparison block), more trials were included for comparison block sizes which were similar to the average height of the standard blocks (i.e. 55mm; comparison block sizes 52-58mm x 10 trials), with fewer trials for comparison blocks which were more dissimilar in size (50 and 60mm x 6 trials, 48 and 
$62 \mathrm{~mm} \times 4$ trials). This amounted to 60 trials per condition (visual, haptic, dual no-conflict, dual conflict standard 1, dual conflict standard 2); 300 trials in total.

Visual only, haptic only and dual conditions were presented separately in blocks of trials. Each block of trials consisted of 30 trials across all comparison block sizes $(52-58 \mathrm{~mm} \times 5$ trials, 50 and $60 \mathrm{~mm} \times 3$ trials, 48 and $62 \mathrm{~mm} \times 2$ trials). This amounted to 10 blocks of trials ( $2 \times$ visual only, $2 \times$ haptic only, 6 $x$ dual). In dual modality conditions, the no-conflict standard and both conflict standards 1 and 2 were intermixed and randomly selected within a block of trials to reduce the chance of participants noticing the conflict manipulation. Three blocks of dual condition trials were always presented in succession to ensure equal presentation of all conflict and no-conflict standards. Participants were given a short break between blocks of trials. The order that blocks of trials was presented was counterbalanced between participants.

\section{Procedure}

Prior to starting the task, the experimenter showed the participant the no-conflict standard and the tallest comparison block, and demonstrated the task procedure. Participants were explicitly told that the blocks were the same on the front and back of the cartridge and represented one single continuous block passing through the cartridge. Participants were also informed that that the depth and length was the same for all blocks; the only dimension that would change between intervals was the height.

Participants were seated in a well-lit room facing the display stand. The seat height was adjusted so that eye-level was approximately aligned with the centre of where the visible blocks would be presented. Participants placed their dominant hand out in front of them on a foam rest at a distance which enabled a comfortable reach around the display stand to where the non-visible (haptic) block would be mounted (see Poole et al., 2017). Participants were instructed to keep their arm out in this position throughout the experiment to ensure that their distance from the visual stimulus remained constant (approximately $40 \mathrm{~cm}$ ). At the start the PLATO spectacles were opaque. A computer screen visually instructed the experimenter which two block sizes would be presented, and in which order. The experimenter selected the cartridge with the first block and mounted it onto the display stand before manually initiating the trial. A voice recording saying "One" signalled the commencement of the trial, and the first interval.

For the haptic only condition, participants were instructed to reach around the display stand and to pinch the block mounted at the rear using their index finger and thumb. Participants were instructed to hold the block for $\sim 1$ second and then to return their hand to its original position. Following the 
completion of the grasp, a break of $2000 \mathrm{~ms}$ allowed enough time for the experimenter to remove the first cartridge from the display stand and to mount the second cartridge displaying the second block. A voice recording saying "Two" then signalled the start of the second interval and for participants to pinch the second haptic block. Participants were required to say which of the intervals ("One" or "Two") contained the taller of the two blocks. The experimenter manually entered the participant's response using the keyboard, which triggered the start of the next trial. The PLATO spectacles remained opaque throughout the haptic only trials.

For the visual only condition, participants were not required to reach towards the haptic block, but instructed to only observe the visible block presented at the front of the display stand. As with the haptic condition, a voice recording of "One" signalled the first interval. After a delay of $500 \mathrm{~ms}$, the PLATO spectacles became transparent for $1000 \mathrm{~ms}$ before turning opaque again. A break of $2000 \mathrm{~ms}$ following the spectacles turning opaque allowed enough time for the experimenter to remove the first cartridge and to display the second, whilst also ensuring that the interval between stimulus presentations was equal to the haptic only trials. A voice recording saying "Two" then signalled the commencement of the second interval. After a delay of $500 \mathrm{~ms}$, the PLATO spectacles became transparent again for $1000 \mathrm{~ms}$ before turning opaque. Participants were again required to say which of the intervals contained the taller of the two blocks.

For the dual visual-haptic condition with and without conflict, the procedure was the same as the visual only condition, except that participants were instructed to also reach towards the haptic block at the rear of the stand after hearing "One". The 500ms delay between the voice signal and the PLATO spectacles becoming transparent allowed enough time for the participant to complete their reach towards the haptic block so that visual and haptic information was presented at approximately the same time. The participant's grasping hand was occluded by the display stand. Participants were instructed to use both vision and touch to judge which interval contained the taller of the two blocks.

Since participants in the study by Gori and colleagues (2008) did not notice the visual-haptic size conflict, it was expected that participants in the current study wouldn't notice the conflict either, and so were not asked whether they noticed the conflict at the end of the experiment. In addition, this study was part of a larger project which included experiments with similar visual-haptic manipulations (see Couth et al., 2018b), and so we did not want to bias participants' perceptions for future studies by questioning them about the conflict. 


\section{Data analysis}

MLE model - Size discrimination thresholds

For each comparison block size, the proportion of trials where the comparison block was judged as taller than the no-conflict standard, conflict standard 1, or conflict standard 2, was calculated. Cumulative Gaussian psychometric functions were then fitted to each condition (visual, haptic, dual no-conflict, dual conflict 1 and dual conflict 2) separately using the Palamedes toolbox (Prins and Kingdom, 2018) in MATLAB R2016a (The MathWorks, Inc., Natick, Massachusetts, United States). Size discrimination thresholds for each condition were determined as the standard deviation (SD) of the Gaussian function (i.e. the inverse of the slope gradient). For the conflict condition, the average of the dual conflict standard 1 and conflict standard 2 thresholds was calculated.

When participants perform close to $100 \%$ accuracy across comparison block sizes in any of the unimodal or multimodal conditions, the psychometric function fit is not reliable, despite showing a good fit to the data ( $p D e v>~ .05$; larger values of pDev indicate better goodness of fit; Kingdom and Prins, 2009), and it is not possible to determine a good estimate of the size discrimination threshold for that condition. In such circumstances, it is not possible to test whether size discrimination thresholds are consistent with MLE. Therefore, participants who performed at $100 \%$ accuracy for more than 6 out of the 8 comparison block sizes, in any condition, were excluded from further analysis (older adults $n=2$, young adults $n=3$ ).

Outliers were identified using the non-recursive procedure described by Van Selst and Jolicoeur (1994), resulting in two outliers (one older and one young) in the visual condition, one outlier (older) in the haptic condition, and three outliers (two older and one young) in dual modality conditions. These outliers were different participants in each condition, and it was not the same participants who were consistently performing poorly across all conditions (i.e. they were not generally struggling to do the task for all conditions). For this reason, we did not remove any outliers or apply any adjustments to these, where removing or altering these data points would affect the calculations of- and comparisons with- the model predictions.

The MLE predicted variance of combined visual-haptic stimuli $\left(\sigma_{v h}^{2}\right)$ is calculated as follows:

$$
\sigma^{2}{ }_{v h}=\frac{\sigma^{2}{ }_{v} \sigma_{h}^{2}}{\sigma^{2}{ }_{v}+\sigma^{2}{ }_{h}} \leq \min \left(\sigma^{2}{ }_{v}, \sigma^{2}{ }_{h}\right)
$$


where $\sigma^{2}$ is the variance of unimodal visual $(v)$ and haptic $(h)$ performance. The MLE variance is smaller than or equal to either of the unimodal variances, reflecting an increase in precision (i.e. the inverse of the variance) in multisensory conditions. The square root of the MLE predicted variance was taken to determine the MLE predicted visual-haptic threshold (i.e. SD).

Shapiro-Wilk tests of normality revealed that size discrimination thresholds were normally distributed for both young and older adults. A repeated measures ANOVA was used to determine whether thresholds differed between all measured (visual, haptic, dual no-conflict and average dual conflict) and model predicted (MLE) thresholds, with age group added as a between subjects factor. Greenhouse-Geisser corrected values are reported when the assumption of sphericity was violated. Main effects were analysed using pairwise comparisons with Bonferroni corrections applied.

To determine the relative strength of evidence to support the null and alternative hypotheses for additional comparisons of interest, Bayesian $t$-tests (independent or paired-samples) with default priors were conducted using JASP (Version 0.9; JASP Team 2018). A Bayes factor $\left(\mathrm{BF}_{10}\right)<1$ suggests increasing evidence for the null over the alternative hypothesis, whereas a $\mathrm{BF}_{10}>1$ suggests increasing evidence for the alternative over the null. A Bayes factor $\left(\mathrm{BF}_{10}\right)>3$ is considered as substantial evidence for the alternative hypothesis, whereas a $\mathrm{BF}_{10}<1 / 3$ is considered as substantial evidence for the null (Dienes, 2014; Rouder et al., 2009).

MLE model - Point of subjective equality (PSE)

The mean of the fitted Gaussian function was used to determine the PSE for each condition (visual, haptic, dual conflict standard 1 and dual conflict standard 2). Conflict standard 1 PSE values were multiplied by -1 to reverse the polarity to match conflict standard 2 (see Poole et al., 2017). The mean average PSE of conflict standard 1 (polarity reversed) and conflict standard 2 was then calculated for each participant. Shapiro-Wilk tests of normality revealed that average measured conflict PSEs were normally distributed for both young and older adults. Two-tailed one-sample $t$ tests were conducted on the average measured conflict PSEs to determine whether there was a significant shift towards visual (positive values) or haptic (negative values) dominance, for each age group separately. A two-tailed independent samples $t$-test compared the average measured conflict PSEs between age groups.

To determine the MLE predicted visual-haptic PSE for conflict conditions, the weight of visual $\left(w_{\mathrm{v}}\right)$ and haptic $\left(w_{h}\right)$ conditions are first calculated separately for each participant:

$$
w_{v}=\frac{\sigma_{h}^{2}}{\sigma_{h}^{2}+\sigma_{v}^{2}}, w_{h}=\frac{\sigma^{2}{ }_{v}}{\sigma_{h}^{2}+\sigma_{v}^{2}}
$$


The MLE predicted PSE $\left(\hat{s}_{v h}\right)$ for no-conflict conditions can be calculated from the weighted sum of the measured PSE for visual $\left(s_{v}\right)$ and haptic $\left(s_{h}\right)$ conditions:

$$
\hat{s}_{v h}=w_{v} s_{v}+w_{h} s_{h}
$$

The MLE of the PSE for dual conflict standard $1\left(\hat{s}_{\text {conflict } 1}\right)$ and dual conflict standard $2\left(\hat{s}_{\text {conflict } 2}\right)$ were therefore calculated in a similar way by substituting the measured unimodal PSEs in equation 3 with the actual size difference of the visual and haptic blocks compared to the no-conflict standard $( \pm 3 \mathrm{~mm})$ :

$$
\hat{\mathrm{s}}_{\text {conflict } 1}=\left(w_{v} \times-3\right)+\left(w_{h} \times 3\right), \quad \hat{\mathrm{s}}_{\text {conflict } 2}=\left(w_{v} \times 3\right)+\left(w_{h} \times-3\right)
$$

Two-tailed paired samples $t$-tests and Pearson's correlations were conducted between predicted conflict MLE PSEs and the average measured conflict standard PSEs, for each age group separately. Bonferroni corrected $p$-values were used to account for multiple comparisons ( $\alpha$ level $=.05 / 5=.01$ ). To determine the relative strength of evidence to support the null and alternative hypotheses for analyses of interest, Bayes Factors were also conducted for each comparison to determine the relative evidence for the alternative or null hypotheses.

Alternative models

Stochastic cue-switching (SCS) model - Size discrimination thresholds

For the SCS model, first the weights for visual and haptic stimuli were calculated as the perturbation of the participant's PSE from a given unisensory cue in the conflict condition, and expressed as a ratio of the overall conflict (see Landy et al., 1995; Young et al., 1993). For example, the visual weight $\left(w_{v}\right)$ for conflict standard 1 is given by:

$$
w_{v}=\frac{c u e_{1}-\mu_{\text {conflict } 1}}{\Delta c u e}
$$

where $\mathrm{cue}_{1}$ refers to the physical properties of the haptic block in conflict standard 1 (i.e. $3 \mathrm{~mm}$ ), $\mu_{\text {conflict } 1}$ is the measured PSE for conflict standard 1 , and $\Delta c u e$ refers to the size of the conflict 
between the stimuli (i.e. $6 \mathrm{~mm}$ ). The visual weight $\left(w_{v}\right)$ therefore represents the extent to which the participant is following the visual stimuli when making size estimates in that conflict condition (up to a maximum of 1 , or minimum of 0$)$. The haptic weight $\left(w_{h}\right)$ for conflict standard 1 is calculated as $1-$ $w_{v}$. For each participant, the mean averages for $w_{v}$ and $w_{h}$ were calculated separately from conflict standard 1 and conflict standard 2.

The variance for the SCS model $\left(\sigma_{v h}^{2}\right)$ was then calculated using these averaged weights and unisensory variances, plus a component due to the discrepancy between unisensory variances:

$$
\sigma_{v h}^{2}=w_{v} \sigma_{v}^{2}+w_{h} \sigma_{h}^{2}+w_{v} w_{h}\left(\mu_{v}-\mu_{h}\right)^{2}
$$

where $\mu$ represents the measured PSEs for visual $(v)$ and haptic $(h)$ stimuli. The square root of the SCS predicted variance was taken to determine the SCS predicted visual-haptic threshold (i.e. SD).

Suboptimal model - Size discrimination thresholds

For the suboptimal model, unisensory cues are integrated, but not weighted according to their reliability. Therefore, the dual modality predicted thresholds are not necessarily reduced compared to unisensory cues (Kuschel et al., 2010). The unisensory cues are combined additively and weighted using the weights calculated in eqn. (5) to give a prediction of dual modality variance $\left(\sigma_{v h}^{2}\right)$ :

$$
\sigma_{v h}^{2}=\left(w_{v} \sigma_{v}\right)^{2}+\left(w_{h} \sigma_{h}\right)^{2}
$$

The square root of the suboptimal predicted variance was taken to determine the suboptimal predicted visual-haptic threshold (i.e. SD).

To determine whether these alternative models predicted dual modality performance, separate analyses were conducted for the SCS and suboptimal predicted thresholds using repeated measures ANOVAs with all measured (visual, haptic, dual no-conflict and average dual conflict) and model predicted (SCS or suboptimal) thresholds included, with age group added as a between subjects factor. Greenhouse-Geisser corrected values are reported when the assumption of sphericity was violated. Main effects were analysed using pairwise comparisons. Bayes Factors were also conducted for additional comparisons of interest to determine the relative evidence for the alternative or null hypotheses. 
Pearson correlation and Bayesian correlation tests were performed between the measured dual modality thresholds and all model predicted thresholds, for conflict and no-conflict conditions, and for each age group separately.

\section{Results}

MLE model - Size discrimination thresholds

[Figure 2.]

The repeated measures ANOVA revealed a main effect of condition $[F(1.900,83.622)=32.595, p<$ $\left..001, \eta_{p}{ }^{2}=.426\right]$. Pairwise comparisons showed that haptic thresholds $(3.44 \mathrm{~mm} \pm .20 \mathrm{~mm})$ were higher than all other conditions (all $p<.001$ ). There were no significant differences between visual $(2.18 \mathrm{~mm} \pm .10 \mathrm{~mm})$, measured dual no-conflict $(2.12 \mathrm{~mm} \pm .10 \mathrm{~mm})$, and average measured dual conflict $(2.37 \mathrm{~mm} \pm .09 \mathrm{~mm})$ thresholds (all $p>.05)$. MLE predicted thresholds $(1.71 \mathrm{~mm} \pm .07 \mathrm{~mm})$ were significantly lower than all other conditions (all $p<.05$ ) (Fig. 2; model 1 ). The main effect of age group $\left[F(1,44)=2.129, p=.152, \eta_{p}^{2}=.046\right]$ and the age group $x$ condition interaction $[F(1.900$, $\left.83.622)=.256, p=.764, \eta_{p}^{2}=.006\right]$ were not significant.

Bayes Factors $\left(\mathrm{BF}_{10}\right)$ were calculated on the comparisons of interest between measured dual noconflict and MLE predicted dual modality thresholds, within each age group. For older adults $\mathrm{BF}_{10}=$ 16.083 indicating that there was strong evidence for the alternative hypothesis (i.e. that dual noconflict and MLE thresholds were drawn from different distributions). For young adults $\mathrm{BF}_{10}=.556$ indicating that there was only anecdotal evidence to support the null hypothesis (i.e. that dual noconflict and MLE thresholds were drawn from the same distribution). Similarly, Bayes Factors were calculated on the comparisons between average measured conflict thresholds and MLE predicted thresholds, within each age group. For older adults $\mathrm{BF}_{10}=1533.064$, and for young adults $\mathrm{BF}_{10}=$ 166.563 , thus indicating that there was very strong evidence for the alternative hypothesis for both age groups (i.e. neither age group was following the MLE model in conflict conditions).

\section{PSE analysis}

Measured visual and haptic weights are shown in Figure 3a. One sample $t$-tests of the mean PSE data revealed significant visual dominance (i.e. PSE shifted towards the visual cue) in the dual conflict condition for older adults [mean PSE $=1.76 \mathrm{~mm} \pm .26 \mathrm{~mm} ; t(27)=6.858, p<.001, d=1.296$ ], but not for younger adults [mean PSE $=.87 \mathrm{~mm} \pm .30 \mathrm{~mm} ; t(17)=2.865, p=.011, d=.675$; Bonferroni corrected $\alpha$ level $=.01]$. For older adults $\mathrm{BF}_{10}=6.974 \times 10^{4}$, indicating extremely strong evidence to support the alternative hypothesis (i.e. visual dominance). For young adults $\mathrm{BF}_{10}=4.990$, indicating 
moderate evidence to support the alternative hypothesis. Mean dual conflict PSEs were not significantly different between young and older adults $[t(44)=2.201, p=.033, d=.665$; Bonferroni corrected $\alpha$ level $=.01]$, and the Bayes Factor revealed limited evidence to support the alternative hypothesis $\left(\mathrm{BF}_{10}=1.992\right)$.

Paired samples $t$-tests showed that average measured dual conflict PSEs were significantly higher than MLE predicted PSEs (i.e. more visual dominance than was predicted) for older adults $[t(27)=$ $3.864, p=.001, d=.730]$ (Fig. 3b), but not for young adults [t(17) $=-.567, p=.578, d=.223$ ] (Fig. 3c). For older adults $\mathrm{BF}_{10}=50.529$, indicating very strong evidence to support the alternative hypothesis, whereas for young adults $\mathrm{BF}_{10}=.280$, indicating moderate evidence to support the null hypothesis. There was a significant correlation between the average conflict PSEs and the MLE predicted conflict PSEs for older adults $(r=.680, \mathrm{n}=28, p<.001)$, but not for young adults $(r=.106, \mathrm{n}=18, p=.675)$.

[Figure 3.]

\section{Alternative models analysis}

SCS model

The repeated measures ANOVA including the SCS model predicted thresholds revealed a main effect of condition $\left[F(2.017,88.754)=21.752, p<.001, \eta_{p}^{2}=.331\right]$. Pairwise comparisons showed that SCS predicted thresholds $(2.51 \mathrm{~mm} \pm .10 \mathrm{~mm})$ were significantly lower than haptic thresholds $(p<.001)$, significantly higher than visual $(p=.001)$ and dual no-conflict thresholds $(p=.043)$, but not significantly different from average dual conflict conditions $(p=.879)$ (Fig. 2; model 2). The main effect of age group $\left[F(1,44)=.880, p=.353, \eta_{p}{ }^{2}=.020\right]$ and the age group $x$ condition interaction $\left[F(2.017,88.754)=.794, p=.456, \eta_{p}^{2}=.018\right]$ were not significant. Bayes Factors were calculated on the comparisons of interest between measured dual conflict and SCS predicted dual modality thresholds, within each age group, indicating only anecdotal evidence to support the null hypothesis in older adults $\left(\mathrm{BF}_{10}=.363\right)$ and moderate evidence to support the alternative hypothesis in young adults $\left(\mathrm{BF}_{10}=4.520\right)$. Comparisons between the average conflict thresholds and the SCS predictions revealed that there was moderate evidence to support the null hypothesis for older adults $\left(\mathrm{BF}_{10}=\right.$ $.210)$, and for young adults there was moderate evidence to support the alternative hypothesis $\left(\mathrm{BF}_{10}\right.$ $=5.276$ ), thus older adults' performance was consistent with the SCS model, whereas young adults' was not.

Suboptimal model 
The repeated measures ANOVA including the suboptimal model predicted thresholds revealed a main effect of condition $\left[F(1.954,85.985)=27.158, p<.001, \eta_{p}{ }^{2}=.382\right]$. Pairwise comparisons showed that suboptimal predicted thresholds $(1.93 \mathrm{~mm} \pm .08 \mathrm{~mm})$ were significantly lower than visual, haptic and average conflict dual modality thresholds (all $p<.05$ ), but not significantly different to dual no-conflict thresholds $(p=1.000$ ) (Fig. 2; model 3). The main effect of age group $\left[F(1,44)=1.526, p=.223, \eta_{p}^{2}=.034\right]$ and the age group $x$ condition interaction $[F(1.954,85.985)=$ $\left..441, p=.640, \eta_{p}^{2}=.010\right]$ were not significant. Comparisons between measured dual no-conflict and suboptimal predicted thresholds showed that there was anecdotal evidence to support the alternative hypothesis for older adults $\left(\mathrm{BF}_{10}=1.300\right)$, whereas for young adults there was moderate evidence to support the null hypothesis $\left(\mathrm{BF}_{10}=.245\right)$. Comparisons between the average conflict thresholds and the suboptimal predictions indicated very strong evidence to support the alternative hypothesis for older adults $\left(\mathrm{BF}_{10}=37.290\right)$. For young adults, there was anecdotal evidence to support the alternative hypothesis $\left(\mathrm{BF}_{10}=1.285\right)$.

Individual participant data for each age group is presented in Fig. 4 with dual modality thresholds plotted as a function of MLE, SCS and suboptimal predicted thresholds, for both conflict and noconflict conditions. There were no significant correlations between any of the model predicted thresholds and measured thresholds, for both age groups, and for both conflicting and nonconflicting conditions (all $p>.05$ ). Bayesian correlations provided moderate evidence to support the null hypothesis for the correlation between dual no-conflict thresholds and sub-optimal thresholds for young adults $\left(\mathrm{BF}_{10}=.303\right)$, and there was moderate evidence to support the null hypothesis for the correlation between dual no-conflict thresholds and SCS thresholds for older adults $\left(\mathrm{BF}_{10}=.279\right)$. All other Bayesian correlations provided weak anecdotal evidence to support the null/alternative hypotheses $\left(.333<\mathrm{BF}_{10}<3\right)$.

\section{[Figure 4.]}

\section{Discussion}

In this study we used a visual-haptic size discrimination task to determine whether the mechanisms of visual-haptic integration are affected by ageing, and whether these mechanisms differ depending on whether visual and haptic cues are equal (non-conflicting) or different (conflicting) in size. Visualhaptic precision did not appear to be affected by ageing on this task, although the mechanisms by which visual-haptic cues were processed may be different for young and older adults, which also varies between individuals. In addition, size discrimination thresholds were similar for both conflicting and non-conflicting stimuli, for both young and older adults. 
For the young adults, visual-haptic thresholds in non-conflicting conditions were more consistent with suboptimal predicted thresholds ${ }^{1}$, and there was some anecdotal evidence to suggest that thresholds were consistent with MLE. On the contrary, older adults' visual-haptic thresholds were not consistent with MLE predicted thresholds, and there was anecdotal evidence that older adults' thresholds were also not consistent with suboptimal thresholds. While there was weak evidence to suggest that older adults may follow a stochastic cue-switching model, no-conflict visual-haptic thresholds were not significantly different to visual thresholds, and a post-hoc Bayesian analysis revealed that there was moderate evidence to support this null hypothesis in older adults $\left(\mathrm{BF}_{10}=\right.$ .204). Therefore, older adults' dual modality performance may be more consistent with visual dominance or 'visual capture', whereby estimations of size are based almost entirely on visual information whilst ignoring haptic information (Rock and Victor, 1964).

When there was a size conflict between visual and haptic cues, measured visual-haptic thresholds were greater than MLE predictions for both young and older adults. For older adults, visual-haptic thresholds were consistent with SCS thresholds. In addition, older adults' PSE data showed significantly more visual dominance than predicted by the MLE model (Fig. 3b). Therefore, it is possible that older adults were relying more exclusively on visual cues (i.e. visual capture) in conflict dual modality conditions also. This tendency for visual dominance in older adults may have been captured by the SCS model, given that SCS dual modality predictions are made using only one of the unisensory cues (usually the more reliable), which may or may not alternate from trial to trial (Kuschel et al., 2010; Landy and Kojima, 2001; Serwe et al., 2009). It is possible that age-related deterioration to the senses means that older adults rely on visual cues to recalibrate or disambiguate other senses (i.e. 'sensory combination'), rather than optimally integrate these (e.g. Gori et al., 2008).

Compared to older adults, young adults' visual-haptic judgements in conflict conditions did not follow SCS predictions. This is also in contrast to our previous study which used a similar paradigm (Poole et al., 2017), where young adults' multisensory performance was more consistent with the SCS model in conflict conditions. Instead, there is some weak evidence to suggest that young adults may integrate visual-haptic cues suboptimally in conflicting conditions also, rather than alternating between the two. This finding supports previous studies that have shown suboptimal integration in young adults when visual and haptic cues lack spatial coincidence (Gepshtein et al., 2005;

\footnotetext{
${ }^{1}$ Note that SCS and suboptimal thresholds are calculated from weights derived from conflict conditions (equation 5), thus some caution should be taken when comparing these alternative models to no-conflict visual-haptic conditions.
} 
Ramkhalawansingh et al., 2018) or vary in size (Billino and Drewing, 2018), whereby it could be less useful, and potentially costly (e.g. error, fatigue, effort) for the central nervous system to integrate confusing conflicting cues in a way that minimises the variance of the multisensory estimate (Rahnev and Denison, 2018).

Overall, the results suggest that, at the group level, young and older adults may use different mechanisms for processing visual-haptic cues. Crucially, this did not have a significant impact on the precision of older adults' size judgements in this simple visual-haptic task. Nonetheless, older adults' over-reliance on visual cues may not be beneficial for more complex tasks which incorporate more sensory (e.g. vision, touch, vestibular, proprioceptive) and/or cognitive resources, such as balance and posture related activities (Anson and Jeka, 2010; Mahoney et al., 2014a; Newell et al., 2011; Setti et al., 2011a; Stapleton et al., 2014). Equally, all of the older adults in the current study were cognitively and physically healthy, whereas relying exclusively on visual stimuli might be detrimental to performance in older adults with some degree of clinically significant cognitive decline and/or general motor or balance impairments (Anson and Jeka, 2010).

At the individual level, there were no significant correlations between dual modality thresholds and model predicted thresholds (MLE, SCS or suboptimal; Fig. 4), for either conflict or no-conflict conditions, for both young and older adults. This finding could suggest that multisensory performance is not predicted by any one model, and suggests that there is considerable between subject variability as to the mechanisms used for dual modality processing (Alais and Burr, 2004; Arnold et al., 2019; Oruç et al., 2003). This finding could highlight the need to assess the mechanisms of multisensory integration at the individual level in order to tailor therapeutic aids and interventions aimed at assisting older adults with everyday activities. For example, Mahoney and colleagues (2014b) have suggested developing visual-tactile stimulators to assist older adults with crossing the street, which could be adapted for each person depending on whether visual or tactile stimuli is more reliable for them. Brooks and colleagues (2015) demonstrated this concept in their audiovisual temporal rate discrimination task, showing that both young and older adults optimally integrated audiovisual cues if the perceptibility of the auditory stimulus was individually adjusted for each older adult. Alternatively, given that previous studies have shown that experienced observers are more likely to follow MLE (e.g. Ernst and Banks, 2002), and given that the ageing brain maintains some plasticity (Burke and Barnes, 2006; Grady, 2012; Gutchess, 2014; Park and Bischof, 2013), this could suggest that older adults could be trained to be flexible in the mechanisms of integration, such as optimally integrating non-conflicting stimuli, and suboptimally integrating or relying on one modality for conflicting stimuli. For example, there is evidence to suggest that training exercises can 
improve multisensory temporal processing in older adults (Merriman et al., 2015; Mozolic et al., 2011; Setti et al., 2014), whilst other training programmes have demonstrated that manipulating visual, vestibular and somatosensory inputs can improve postural stability (Anson and Jeka, 2010; Hu and Woollacott, 1994a, 1994b). It may be feasible to advance on these training programmes by training older adults to optimally integrate sensory cues for posture and balance.

What remains unclear from the current experiment is why participants did not optimally integrate non-conflicting visual-haptic stimuli, considering that our study was closely modelled on that by Gori and colleagues (2008) who were able to demonstrate optimal integration in young adults. Rahnev and Denison (2018) suggest that task demands and/or prior expectations about whether cues should be integrated or not may encourage participants to adopt an alternative strategy. In the current experiment all participants were inexperienced psychological observers and had no prior knowledge of the task, thus performance may have been more variable and may be influenced by non-sensory factors, such as which decision making strategy participants adopted (Jakel and Wichmann, 2006). In contrast, the young adults in the study by Gori and colleagues (2008) were a small sample $(n=3)$ who were well trained on the task before collecting the data. Alternatively, it is possible that intermixing conflicting and non-conflicting conditions increased awareness of the size conflict (the opposite of what was intended), which forced participants to use a different mechanism of integration (i.e. suboptimal, visual capture or SCS) or different decision making strategy, for both conflicting and non-conflicting conditions. In addition, participants may have been less capable of dividing attention between vision and touch, especially for older adults (for a review of divided attention in ageing, see Glisky, 2007) or for those who were aware of the conflict. Consequently, this could have made it difficult to form an optimally weighted visual-haptic estimate in non-conflicting conditions, and could have also encouraged participants to focus on one modality (i.e. visual capture). It is crucial for future experiments to ascertain whether participants noticed the size conflict or not in order to test these hypotheses (Gori et al., 2008). Future experiments might also consider separating conflicting and non-conflicting dual modality conditions to better clarify whether the mechanisms of integration differ between these conditions, and how this might be affected by ageing.

It is important to highlight that our previous study which used a similar paradigm also did not show optimal integration (Poole et al., 2017), which could question the suitability of this task for investigating integration, even for no-conflict conditions. Similar to how participants in the study by Billino and Drewing (2018) may have relied on haptic only cues in visual-haptic conditions; participants in the current experiment may have relied on visual only cues in visual-haptic 
conditions. Indeed, haptic thresholds were worse than all other conditions, and visual thresholds were not significantly different to conflict and no-conflict dual modality thresholds (Fig. 2), suggesting there may have been little opportunity to benefit from multisensory cues; the most multisensory benefit should be observed when unisensory thresholds are equal (Ernst and Banks, 2002). Nevertheless, the PSE data (Fig. 3b and c) shows that although there is a tendency towards reliance on visual cues, there are still a large number of participants who are using at least some haptic information to make their size judgements, and some participants who perform close to optimal (see also Figure 7 in Poole et al., 2017). This suggests that the task is suitable for capturing optimal integration (as was also shown by Gori et al., 2008), as well as alternative models of multisensory processing (e.g. SCS and suboptimal). However, it may be better to assess integration at the individual level, whereby larger and more heterogeneous groups are more variable in the strategy used, which could make it difficult to capture performance at the group level (cf. Gori et al., 2008).

In conclusion, the current study suggests that the ability to make size judgements using visual-haptic cues is similar for young and older adults. However, the data may suggest that the mechanism used to achieve these judgements is different for each age group. Young adults tended to follow a suboptimal model of integration to process conflicting and non-conflicting visual-haptic size information. Conversely, older adults tended to rely more exclusively on visual cues to make visualhaptic size judgements for both equal and conflicting stimuli. Whilst the older adults' strategy may suffice for simple bimodal size discrimination tasks, it may be less effective for more complex activities involving more cognitive and sensory resources. This has implications for developing preventative or rehabilitative aids which promote the up-weighting of reliable sensory cues, which needs to be tailored to the needs of the individual.

\section{Acknowledgements}

This research was funded by the Frederick Craven Moore Studentship Award; The University of Manchester.

\section{References}

Alais, D., Burr, D., 2004. The Ventriloquist Effect Results from Near-Optimal Bimodal Integration. Curr. Biol. 14, 257-262. doi:10.1016/j.cub.2004.01.029 
Anson, E., Jeka, J., 2010. Sensory reweighting: a rehabilitative mechanism, in: Armstrong, C.L., Morrow, L. (Eds.), Handbook of Medical Neuropsychology: Applications of Cognitive Neuroscience. Springer Science + Business Media; US, New York, NY, US, pp. 519-529.

Arnold, D.H., Petrie, K., Murray, C., Johnston, A., 2019. Suboptimal human multisensory cue combination. Sci. Rep. 9, 5155. doi:10.1038/s41598-018-37888-7

Bates, S.L., Wolbers, T., 2014. How cognitive aging affects multisensory integration of navigational cues. Neurobiol. Aging 35, 2761-2769. doi:10.1016/j.neurobiolaging.2014.04.003

Berg, K., 1989. Measuring balance in the elderly: preliminary development of an instrument. Physiother. Canada 41, 304-311. doi:10.3138/ptc.41.6.304

Billino, J., Drewing, K., 2018. Age Effects on Visuo-Haptic Length Discrimination: Evidence for Optimal Integration of Senses in Senior Adults. Multisens. Res. 31, 273-300. doi:https://doi.org/10.1163/22134808-00002601

Braem, B., Honoré, J., Rousseaux, M., Saj, A., Coello, Y., 2014. Integration of visual and haptic informations in the perception of the vertical in young and old healthy adults and right braindamaged patients. Neurophysiol. Clin. Neurophysiol. 44, 41-48. doi:10.1016/j.neucli.2013.10.137

Brooks, C.J., Anderson, A.J., Roach, N.W., McGraw, P. V, McKendrick, A.M., 2015. Age-related changes in auditory and visual interactions in temporal rate perception. J. Vis. 15, 2. doi:10.1167/15.16.2

Burke, S.N., Barnes, C.A., 2006. Neural plasticity in the ageing brain. Nat. Rev. Neurosci. 7, 30-40. doi:10.1038/nrn1809

Cellini, C., Kaim, L., Drewing, K., 2013. Visual and haptic integration in the estimation of softness of deformable objects. Iperception. 4, 516-31. doi:10.1068/i0598

Cienkowski, K.M., Carney, A.E., 2002. Auditory-visual speech perception and aging. Ear Hear. 23, 439-449. doi:00003446-200210000-00006

Couth, S., Gowen, E., Poliakoff, E., 2018a. Using race model violation to explore multisensory responses in older adults: Enhanced multisensory integration or slower unisensory processing? Multisens. Res. 31. doi:10.1163/22134808-00002588

Couth, S., Gowen, E., Poliakoff, E., 2018b. How does ageing affect grasp adaptation to a visual-haptic 
size conflict? Exp. Brain Res. doi:10.1007/s00221-018-5288-1

Couth, S., Gowen, E., Poliakoff, E., 2016. Investigating the spatial and temporal modulation of visuotactile interactions in older adults. Exp. Brain Res. 234. doi:10.1007/s00221-015-4431-5

de Dieuleveult, A.L., Siemonsma, P.C., van Erp, J.B.F., Brouwer, A.-M., 2017. Effects of Aging in Multisensory Integration: A Systematic Review. Front. Aging Neurosci. 9, 80. doi:10.3389/fnagi.2017.00080

De Gelder, B., Bertelson, P., 2003. Multisensory integration, perception and ecological validity. Trends Cogn. Sci. 7, 460-467. doi:10.1016/j.tics.2003.08.014

Dienes, Z., 2014. Using Bayes to get the most out of non-significant results. Front. Psychol. 5, 781. doi:10.3389/fpsyg.2014.00781

Drewing, K., Ernst, M.O., 2006. Integration of force and position cues for shape perception through active touch. Brain Res. 1078, 92-100. doi:10.1016/j.brainres.2005.12.026

Dumas, K., Holtzer, R., Mahoney, J.R., 2016. Visual-Somatosensory Integration in Older Adults: Links to Sensory Functioning. Multisens. Res. 29, 397-420. doi:10.1163/22134808-00002521

Eikema, D.J.A., Hatzitaki, V., Tzovaras, D., Papaxanthis, C., 2012. Age-dependent modulation of sensory reweighting for controlling posture in a dynamic virtual environment. Age (Dordr). 34, 1381-92. doi:10.1007/s11357-011-9310-9

Ernst, M.O., Banks, M.S., 2002. Humans integrate visual and haptic information in a statistically optimal fashion. Nature 415, 429-433. doi:415429a [pii] 10.1038/415429a

Ernst, M.O., Bülthoff, H.H., 2004. Merging the senses into a robust percept. Trends Cogn. Sci. 8, 162169. doi:10.1016/j.tics.2004.02.002

Falkenstein, I.A., Cochran, D.E., Azen, S.P., Dustin, L., Tammewar, A.M., Kozak, I., Freeman, W.R., 2008. Comparison of visual acuity in macular degeneration patients measured with snellen and early treatment diabetic retinopathy study charts. Ophthalmology 115, 319-23. doi:10.1016/j.ophtha.2007.05.028

Folstein, M.F., Folstein, S.E., McHugh, P.R., 1975. "Mini-mental state”. A practical method for grading the cognitive state of patients for the clinician. J. Psychiatr. Res. 12, 189-98. doi:10.1016/00223956(75)90026-6

Gepshtein, S., Burge, J., Ernst, M.O., Banks, M.S., 2005. The combination of vision and touch depends 
on spatial proximity. J Vis 5, 1013-1023. doi:/5/11/7/ [pii] 10.1167/5.11.7

Glisky, E.L., 2007. Changes in Cognitive Function in Human Aging, Brain Aging: Models, Methods, and Mechanisms. CRC Press/Taylor \& Francis.

Gori, M., Del Viva, M., Sandini, G., Burr, D.C., 2008. Young children do not integrate visual and haptic form information. Curr. Biol. 18, 694-8. doi:10.1016/j.cub.2008.04.036

Grady, C., 2012. The cognitive neuroscience of ageing. Nat. Rev. Neurosci. 13, 491-505. doi:10.1038/nrn3256

Gutchess, A., 2014. Plasticity of the aging brain: New directions in cognitive neuroscience. Science (80-. ). 346, 579-582. doi:10.1126/SCIENCE.1254604

Hillis, J.M., Watt, S.J., Landy, M.S., Banks, M.S., 2004. Slant from texture and disparity cues: Optimal cue combination. J. Vis. 4, 1. doi:10.1167/4.12.1

Hu, M.H., Woollacott, M.H., 1994a. Multisensory training of standing balance in older adults: I. Postural stability and one-leg stance balance. J. Gerontol. 49, M52-61. doi:10.1093/geronj/49.2.M52

Hu, M.H., Woollacott, M.H., 1994b. Multisensory training of standing balance in older adults: II. Kinematic and electromyographic postural responses. J. Gerontol. 49, M62-71. doi:10.1093/geronj/49.2.M62

Hugenschmidt, C., Mozolic, J., Laurienti, P., 2009. Suppression of multisensory integration by modality-specific attention in aging. Neuroreport 20, 349-353. doi:10.1097/WNR.0b013e328323ab07

Jakel, F., Wichmann, F.A., 2006. Spatial four-alternative forced-choice method is the preferred psychophysical method for naive observers. J. Vis. 6, 13-13. doi:10.1167/6.11.13

Jeka, J., Allison, L., Saffer, M., Zhang, Y., Carver, S., Kiemel, T., 2006. Sensory reweighting with translational visual stimuli in young and elderly adults: the role of state-dependent noise. Exp Brain Res 174, 517-527. doi:10.1007/s00221-006-0502-y

Kaiser, P.K., 2009. Prospective evaluation of visual acuity assessment: a comparison of snellen versus ETDRS charts in clinical practice (An AOS Thesis). Trans. Am. Ophthalmol. Soc. 107, 311-24.

Kingdom, F.A.A., Prins, N., 2009. Psychophysics: A Practical Introduction. Academic Press, London. 
Kuschel, M., Di Luca, M., Buss, M., Klatzky, R.L., 2010. Combination and Integration in the Perception of Visual-Haptic Compliance Information. IEEE Trans. Haptics 3, 234-244. doi:10.1109/ТОН.2010.9

Landy, M.S., Kojima, H., 2001. Ideal cue combination for localizing texture-defined edges. J. Opt. Soc. Am. A. Opt. Image Sci. Vis. 18, 2307-20.

Landy, M.S., Maloney, L.T., Johnston, E.B., Young, M., 1995. Measurement and modeling of depth cue combination: in defense of weak fusion. Vision Res. 35, 389-412. doi:10.1016/00426989(94)00176-M

Laurienti, P.J., Burdette, J.H., Maldjian, J.A., Wallace, M.T., 2006. Enhanced multisensory integration in older adults. Neurobiol. Aging 27, 1155-1163. doi:10.1016/j.neurobiolaging.2005.05.024

Mahboobin, A., Loughlin, P.J., Redfern, M.S., Sparto, P.J., 2005. Sensory re-weighting in human postural control during moving-scene perturbations. Exp. Brain Res. 167, 260-7. doi:10.1007/s00221-005-0053-7

Mahoney, J.R., Dumas, K., Holtzer, R., 2015. Visual-Somatosensory Integration is Linked to Physical Activity Level in Older Adults. Multisens. Res. 28. doi:10.1163/22134808-00002470

Mahoney, J.R., Holtzer, R., Verghese, J., 2014a. Visual-somatosensory integration and balance: evidence for psychophysical integrative differences in aging. Multisens. Res. 27, 17-42. doi:10.1163/22134808-00002444

Mahoney, J.R., Li, P.C.C., Oh-Park, M., Verghese, J., Holtzer, R., 2011. Multisensory integration across the senses in young and old adults. Brain Res. 1426, 43-53. doi:10.1016/j.brainres.2011.09.017

Mahoney, J.R., Wang, C., Dumas, K., Holtzer, R., 2014b. Visual-somatosensory integration in aging: Does stimulus location really matter? Vis. Neurosci. 31, 275-283. doi:10.1017/S0952523814000078

Merriman, N.A., Whyatt, C., Setti, A., Craig, C., Newell, F.N., 2015. Successful balance training is associated with improved multisensory function in fall-prone older adults. Comput. Human Behav. 45, 192-203. doi:10.1016/j.chb.2014.12.017

Mozolic, J., Hugenschmidt, C., Peiffer, A., Laurienti, P., 2012. Multisensory integration and aging, in: Murray, M.M., Wallace, M.T. (Eds.), The Neural Bases of Multisensory Processes. CRC Press, Boca Raton, FL. 
Mozolic, J.L., Long, A.B., Morgan, A.R., Rawley-Payne, M., Laurienti, P.J., 2011. A cognitive training intervention improves modality-specific attention in a randomized controlled trial of healthy older adults. Neurobiol. Aging 32, 655-68. doi:10.1016/j.neurobiolaging.2009.04.013

Newell, F.N., Setti, A., Foran, T.G., Burke, K., Kenny, R.A., 2011. Reduced vision impairs spatial cognition in fall-prone older adults. Insight Res. Pract. Vis. Impair. Blind. 4, 103-111.

Nusbaum, N.J., 1999. Aging and sensory senescence. South. Med. J. 92, 267-275. doi:10.1097/00007611-199903000-00002

Oruç, I., Maloney, L.T., Landy, M.S., 2003. Weighted linear cue combination with possibly correlated error. Vision Res. 43, 2451-68.

Park, D.C., Bischof, G.N., 2013. The aging mind: neuroplasticity in response to cognitive training. Dialogues Clin. Neurosci. 15, 109-19.

Peiffer, A.M., Mozolic, J.L., Hugenschmidt, C.E., Laurienti, P.J., 2007. Age-related multisensory enhancement in a simple audiovisual detection task. Neuroreport 18, 1077-81. doi:10.1097/WNR.0b013e3281e72ae7

Poliakoff, E., Ashworth, S., Lowe, C., Spence, C., 2006a. Vision and touch in ageing: crossmodal selective attention and visuotactile spatial interactions. Neuropsychologia 44, 507-17. doi:10.1016/j.neuropsychologia.2005.07.004

Poliakoff, E., Shore, D.I., Lowe, C., Spence, C., 2006b. Visuotactile temporal order judgments in ageing. Neurosci. Lett. 396, 207-211. doi:10.1016/j.neulet.2005.11.034

Poole, D., Poliakoff, E., Gowen, E., Couth, S., Champion, R.A., Warren, P.A., 2017. Similarities in autistic and neurotypical visual-haptic perception when making judgements about conflicting sensory stimuli. Multisens. Res. 30. doi:10.1163/22134808-00002551

Prins, N., Kingdom, F.A.A., 2018. Applying the Model-Comparison Approach to Test Specific Research Hypotheses in Psychophysical Research Using the Palamedes Toolbox. Front. Psychol. 9, 1250. doi:10.3389/fpsyg.2018.01250

Rahnev, D., Denison, R.N., 2018. Suboptimality in perceptual decision making. Behav. Brain Sci. 41, e223. doi:10.1017/S0140525X18000936

Ramkhalawansingh, R., Butler, J.S., Campos, J.L., 2018. Visual-vestibular integration during selfmotion perception in younger and older adults. Psychol. Aging 33, 798-813. 
doi:10.1037/pag0000271

Ravaioli, E., Oie, K.S., Kiemel, T., Chiari, L., Jeka, J.J., 2005. Nonlinear postural control in response to visual translation. Exp. Brain Res. 160, 450-9. doi:10.1007/s00221-004-2030-y

Rock, I., Victor, J., 1964. Vision and touch: An experimentally created conflict between the two senses. Science 143, 594-6. doi:10.1126/science.143.3606.594

Rohde, M., van Dam, L.C.J., Ernst, M., 2016. Statistically Optimal Multisensory Cue Integration: A Practical Tutorial. Multisens. Res. 29, 279-317.

Rosas, P., Wagemans, J., Ernst, M.O., Wichmann, F.A., 2005. Texture and haptic cues in slant discrimination: reliability-based cue weighting without statistically optimal cue combination. J. Opt. Soc. Am. A. Opt. Image Sci. Vis. 22, 801-9.

Rouder, J.N., Speckman, P.L., Sun, D., Morey, R.D., Iverson, G., 2009. Bayesian t tests for accepting and rejecting the null hypothesis. Psychon. Bull. Rev. 16, 225-237. doi:10.3758/PBR.16.2.225

Serwe, S., Drewing, K., Trommershauser, J., 2009. Combination of noisy directional visual and proprioceptive information. J. Vis. 9, 28-28. doi:10.1167/9.5.28

Setti, A., Burke, K., Kenny, R., Newell, F., 2011a. Is inefficient multisensory processing associated with falls in older people? Exp. Brain Res. 209, 375-384. doi:10.1007/s00221-011-2560-z

Setti, A., Finnigan, S., Sobolewski, R., McLaren, L., Robertson, I.H., Reilly, R.B., Kenny, R.A., Newell, F.N., 2011b. Audiovisual temporal discrimination is less efficient with aging: an event-related potential study. Neuroreport 22, 554-8. doi:10.1097/WNR.0b013e328348c731

Setti, A., Stapleton, J., Leahy, D., Walsh, C., Kenny, R.A., Newell, F.N., 2014. Improving the efficiency of multisensory integration in older adults: audio-visual temporal discrimination training reduces susceptibility to the sound-induced flash illusion. Neuropsychologia 61, 259-68. doi:10.1016/j.neuropsychologia.2014.06.027

Stapleton, J., Setti, A., Doheny, E.P., Kenny, R.A., Newell, F.N., 2014. A standing posture is associated with increased susceptibility to the sound-induced flash illusion in fall-prone older adults. Exp. Brain Res. 232, 423-34. doi:10.1007/s00221-013-3750-7

Stein, B.E., Meredith, M.A., 1993. The Merging of the Senses. MIT Press.

Sundermier, L., Woollacott, M.H., Jensen, J.L., Moore, S., 1996. Postural sensitivity to visual flow in aging adults with and without balance problems. J Gerontol A Biol Sci Med Sci 51, 45-52. 
doi:10.1093/gerona/51A.2.M45

Teramoto, W., Honda, K., Furuta, K., Sekiyama, K., 2017. Visuotactile interaction even in far sagittal space in older adults with decreased gait and balance functions. Exp. Brain Res. 1-15. doi:10.1007/s00221-017-4975-7

Van Dam, L.C.J., Parise, C. V., Ernst, M.O., Bennett, D.J., Hill, C.S., 2014. Modeling multisensory integration, in: Bennett, D., Hill, C. (Eds.), Sensory Integration and the Unity of Consciousness. MIT Press, pp. 209-229.

Van Selst, M., Jolicoeur, P., 1994. A solution to the effect of sample size on outlier elimination. Q. J. Exp. Psychol. Sect. A 47, 631-650. doi:10.1080/14640749408401131

Virsu, V., Lahti-Nuuttila, P., Laasonen, M., 2003. Crossmodal temporal processing acuity impairment aggravates with age in developmental dyslexia. Neurosci. Lett. 336, 151-154. doi:10.1016/S0304-3940(02)01253-3

Young, M.J., Landy, M.S., Maloney, L.T., 1993. A perturbation analysis of depth perception from combinations of texture and motion cues. Vision Res. 33, 2685-2696. doi:10.1016/00426989(93)90228-0 


\section{Figure captions}

Figure 1. Apparatus showing a) the standard no-conflict block $(55 \mathrm{~mm})$ plus comparison block sizes, b) the conflict standard blocks, where the forward-facing visual block is either shorter (conflict standard 1) or taller (conflict standard 2), and c) the switching of the cartridges which display the blocks from the display stand. Colour images available online.

Figure 2. Mean size discriminaton thresholds ( \pm SEM) for older (blue diagonal lines) and young (red hatch marking) participants for all measured conditions (visual, haptic, dual no-conflict and average dual conflict) and model predictions; MLE (model 1), SCS (model 2), and suboptimal (model 3). Colour images available online.

Figure $3 . \quad$ a) visual (solid bars; left vertical axis) and haptic (shaded bars; right vertical axis) weights derived from equation (2) for older (blue) and younger (red) adults. MLE predicted conflict PSEs against average conflict PSEs are also shown for $b$ ) older adults (blue diamonds) and c) young adults (red squares). The dashed $x=y$ line represents where the measured PSEs would lie if conflict visual-haptic PSEs were perfectly modelled by the MLE prediction. The dotted horizontal lines indicate where measured PSEs would lie for complete visual (top) or complete haptic (bottom) dominance. Colour images available online.

Figure 4. Predicted thresholds against measured thresholds for a) older adults, no-conflict conditions, b) young adults, no-conflict conditions, c) older adults, conflict conditions, and d) young adults, conflict conditions. The dashed $x=y$ line represents where the measured points would lie if visual-haptic thresholds were perfectly modelled by the predictions. The coloured lines represent the lines of best fit for MLE (blue; diamonds), SCS (red; squares) and Suboptimal (green; triangles) models. Colour images available online. 
Figure 1

a)

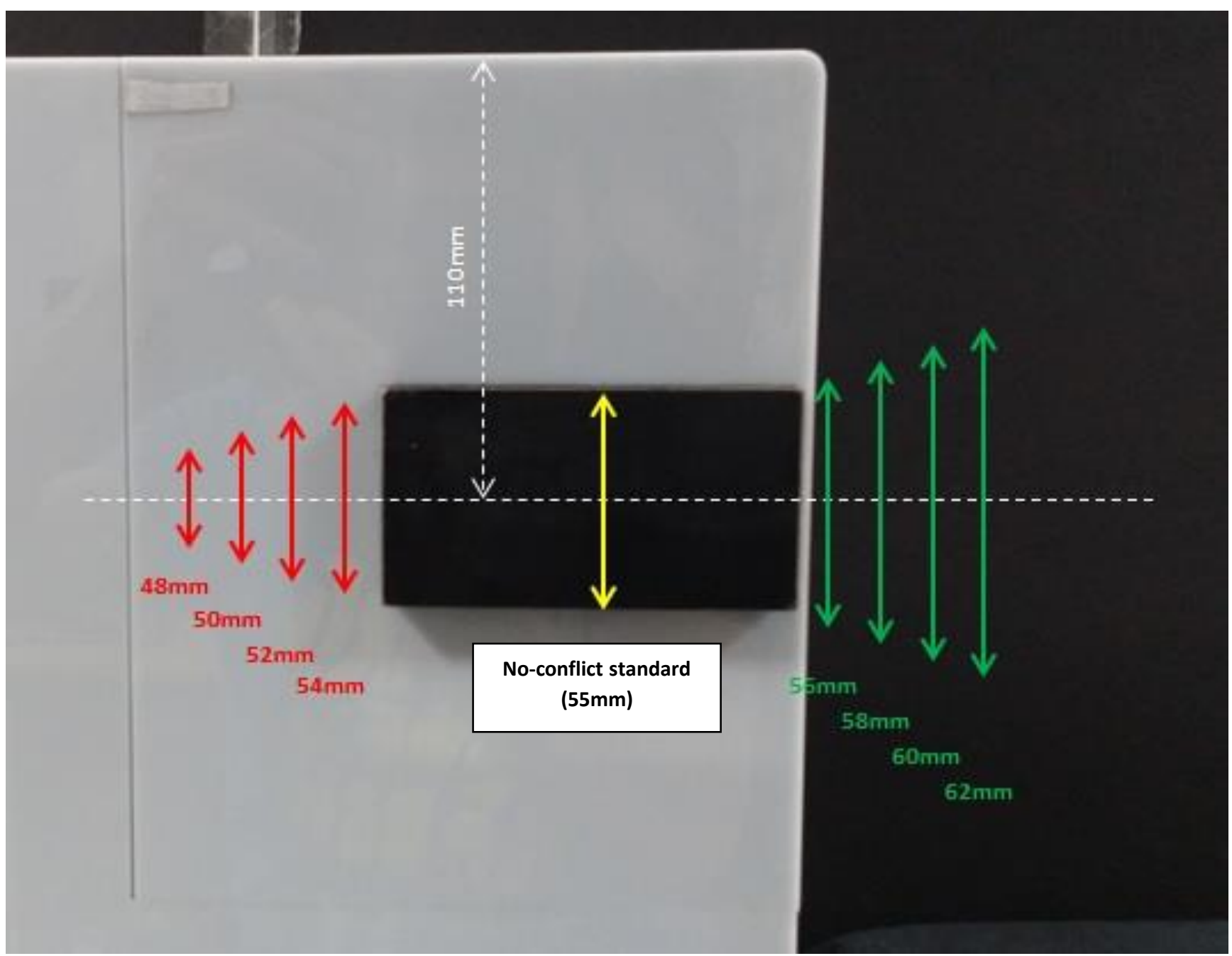

b)

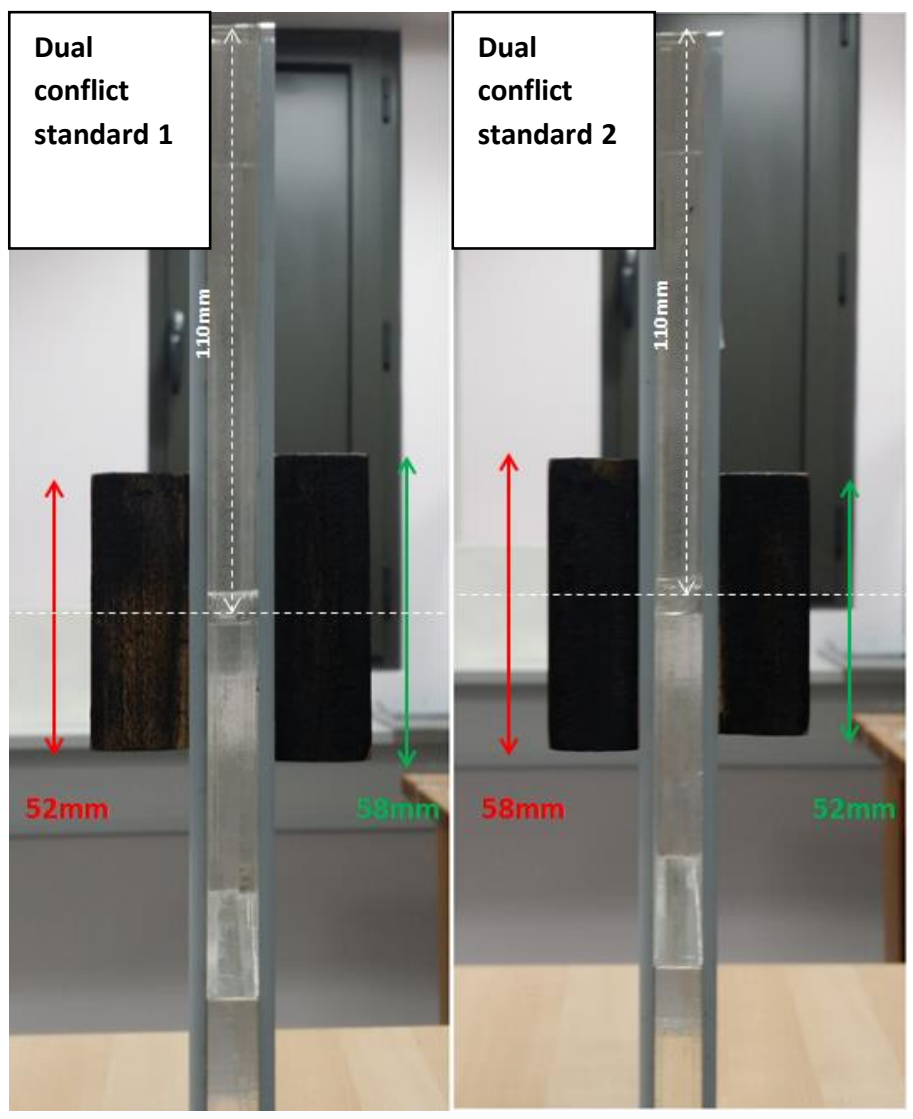

c)

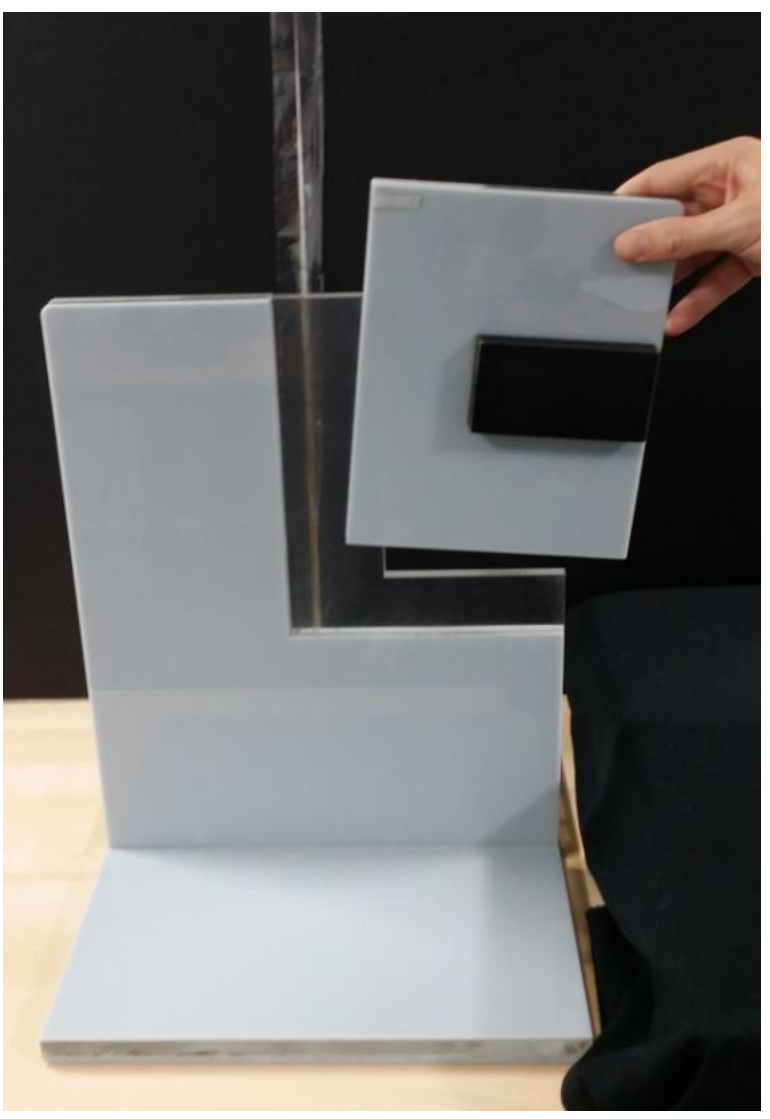


Figure 2

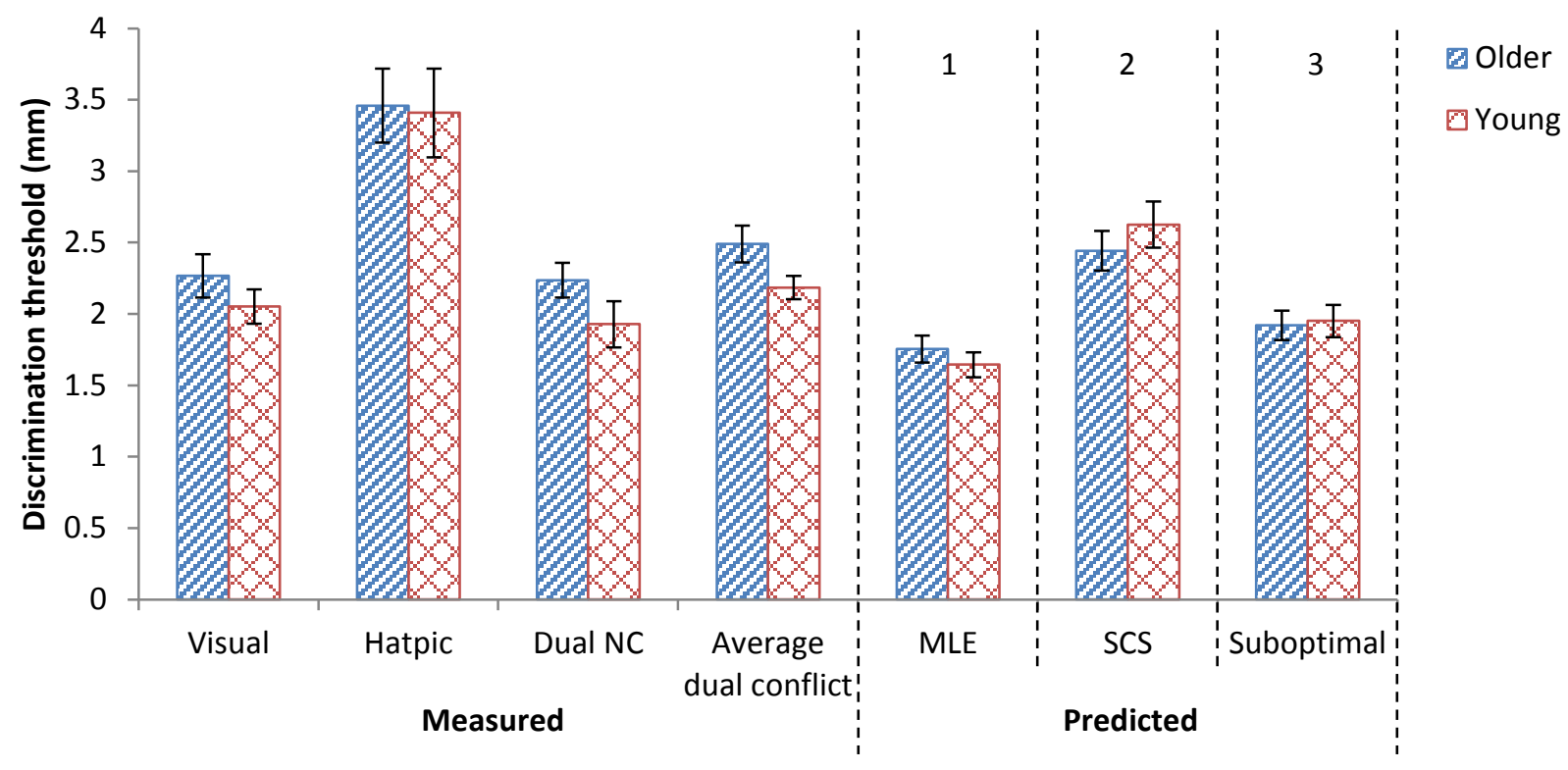


Figure 3

a)

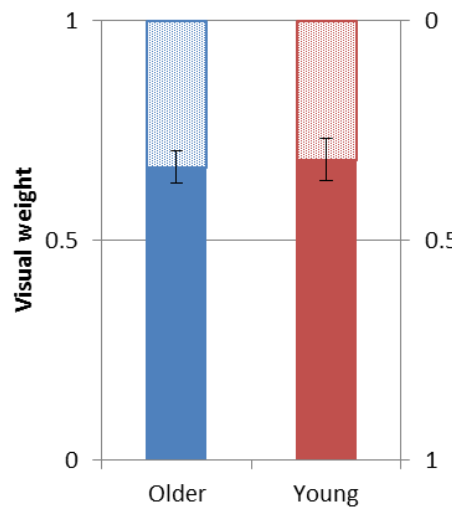

b)

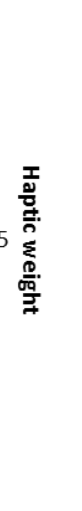

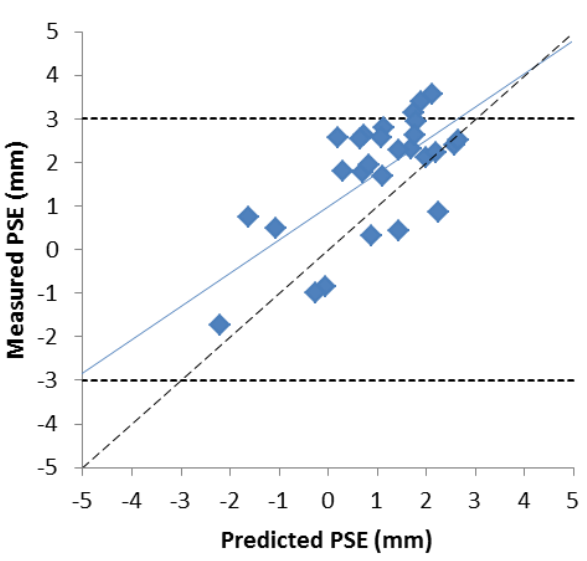

c)

Young

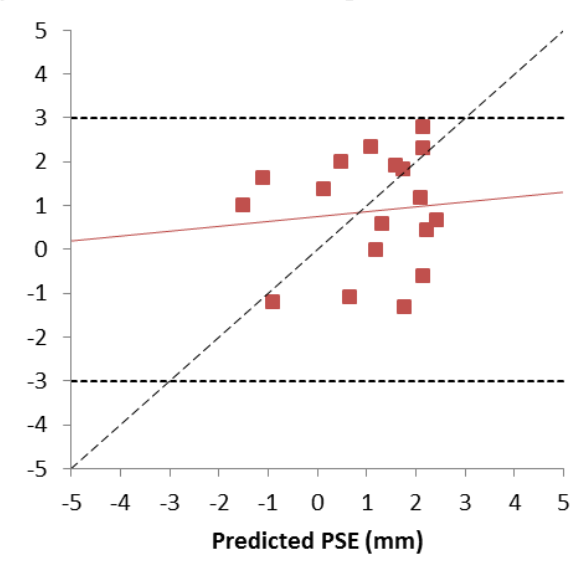




\section{Figure 4}

a)

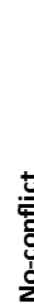

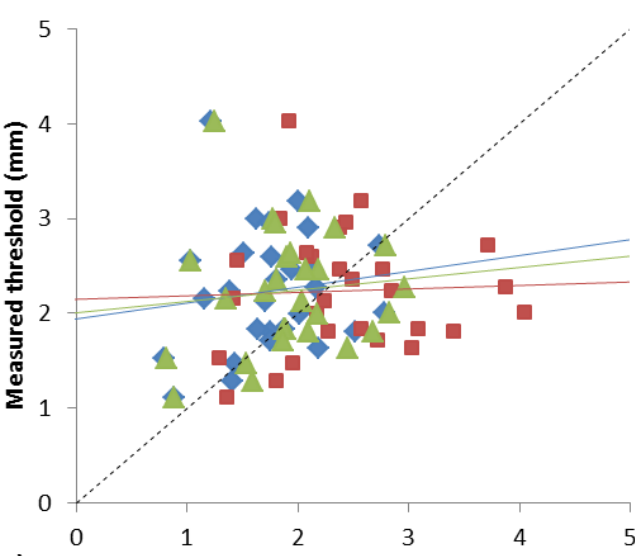

c)

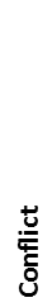

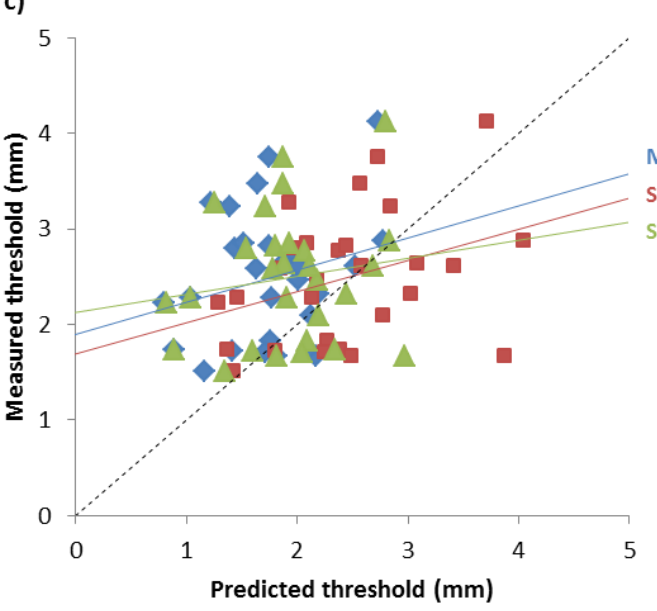

$\operatorname{MLE}(r=.129, p=.512)$

Subop $(r=.103, p=.604)$

$\operatorname{SCS}(r=.042, p=.833)$

b)

Young
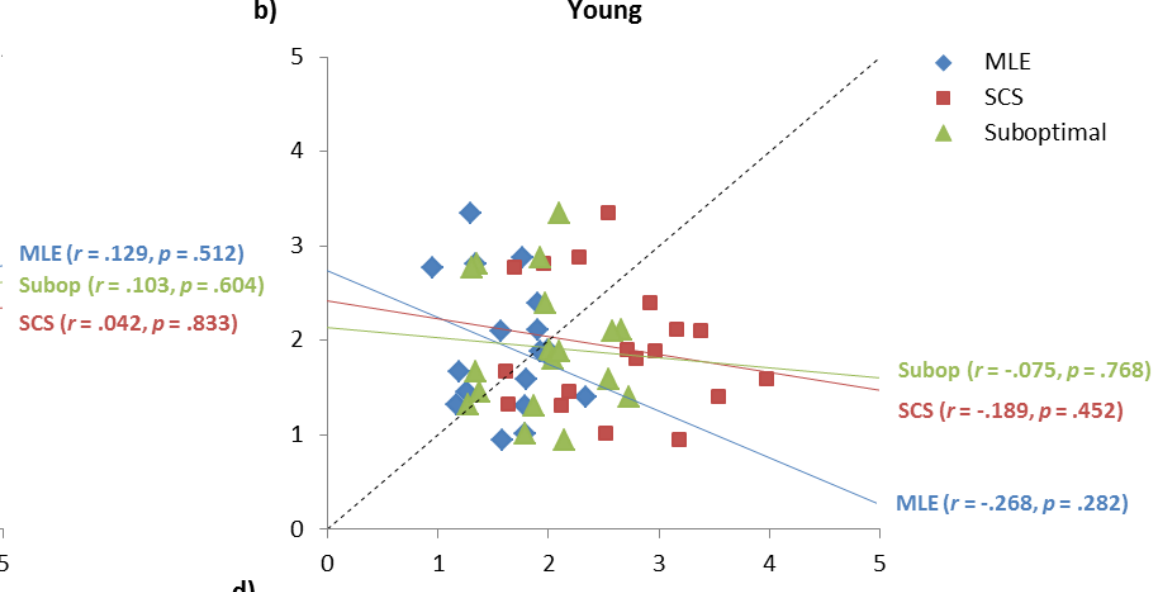

d)

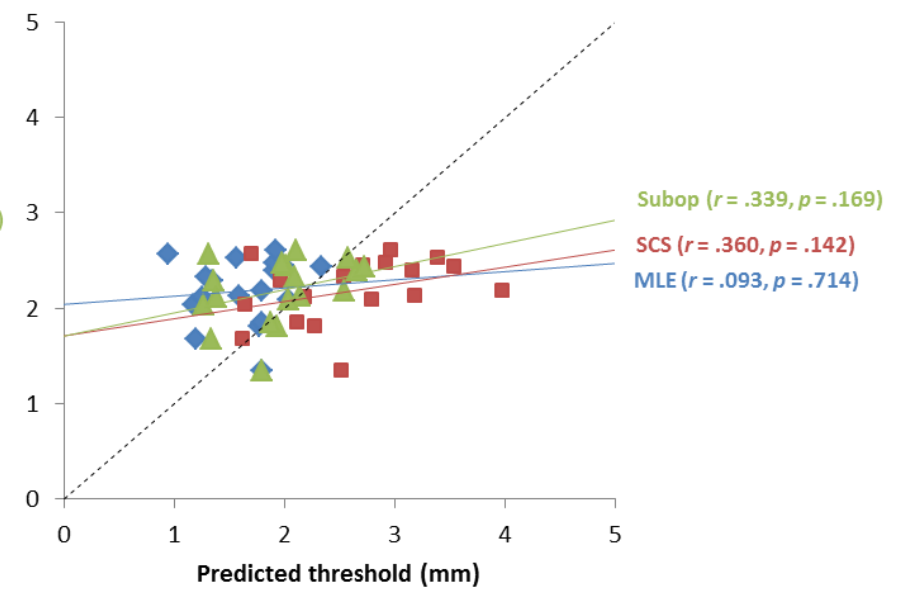

